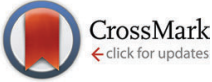

Cite this: Phys. Chem. Chem. Phys., 2016, 18, 25036

Received 15th June 2016, Accepted 10th August 2016

DOI: $10.1039 / c 6 c p 04193 k$

www.rsc.org/pccp

\section{Influence of counterions on the conformation of conjugated polyelectrolytes: the case of poly(thiophen-3-ylacetic acid) $\uparrow$}

\author{
Gregor Hostnik, ${ }^{a}$ Matjaž Bončina, ${ }^{a}$ Caterina Dolce, ${ }^{b}$ Guillaume Mériguet, ${ }^{b}$ \\ Anne-Laure Rollet ${ }^{\mathrm{b}}$ and Janez Cerar*a
}

\begin{abstract}
The addition of simple salt to a solution of conjugated polyelectrolyte can lead to substantial changes in its optical properties caused by the conformational change of the polymer chain. The effect of the addition of alkali metal and tetraalkylammonium chlorides to solutions of lithium salt of poly(thiophen-3-ylacetic acid) is investigated by NMR. The fractions of free alkali metal counterions are in agreement with predictions of the cylindrical Poisson-Boltzmann cell model. On the other hand, the fractions of free tetraalkylammonium counterions deviate from the prediction of this model and diminish with increasing size of these counterions. This trend is consistent with observed ultraviolet-visible absorption spectra and measured self-diffusion coefficients of the polyion in mixtures with tetraalkylammonium salts. A transition to more constricted conformation of the polyion chain becomes more pronounced with the lengthening of alkyl groups in the added tetraalkylammonium cation. Taking into account the obtained fractions of free counterions, existing thermodynamic data are reanalysed in order to determine thermodynamic parameters for binding of different counterions to the polyion. This analysis shows that standard enthalpies of binding of alkali metal counterions are distinctively different, which is most probably related to differences in hydration shells of counterions. On the other hand, such an analysis fails in the case of tetraalkylammonium chlorides where obviously more complex changes take place.
\end{abstract}

\section{Introduction}

Poly(thiophen-3-ylacetic acid) (PTAA) is a polymer (Fig. 1) from a class of conjugated polyelectrolytes (PEs). These polymers are, due to their favourable physical properties, the subject of intensive application studies in the field of optoelectronic devices $^{1-9}$ and sensors. ${ }^{10-24}$ Although conjugated PEs are mostly used in the form of thin layers, ${ }^{1-9}$ their properties in solutions, from which those layers are made, are of crucial importance. ${ }^{25-27}$ One of the parameters that have a decisive effect on the properties of conjugated PEs in the solid state ${ }^{7,25}$ and in solution ${ }^{28}$ is the nature of the counterion.

It is well known that the spectral properties of conjugated PE solutions depend on their conformation and the potential formation of aggregates. ${ }^{28-30}$ Both properties can be influenced by the degree of neutralisation and by the selection of counterion species. In this respect, polythiophenes are no exception.

\footnotetext{
${ }^{a}$ Faculty of Chemistry and Chemical Technology, University of Ljubljana, Večna pot 113,SI-100o Ljubljana, Slovenia.E-mail: janez.cerar@fkkt.uni-lj.si

${ }^{b}$ Sorbonne Universités, UPMC Univ Paris 06, CNRS, Laboratoire PHENIX, Paris, France

† Electronic supplementary information (ESI) available. See DOI: 10.1039/c6cp04193k
}

Kim et $a l .{ }^{29}$ and Vallat et $a l .{ }^{30}$ have shown that the position of the absorption maximum in the spectra of PTAA aqueous solutions depends on the level of neutralisation. In both cases a red shift was observed when the degree of neutralisation was increased. This effect was explained by the conformational change induced by the increased density of charged groups on the polyion. ${ }^{29,30}$

An existence of the ionochromic effect in polythiophene solutions was observed by McCullough et al. ${ }^{28}$ who found a strong dependence of absorption spectra on the counterion nature.

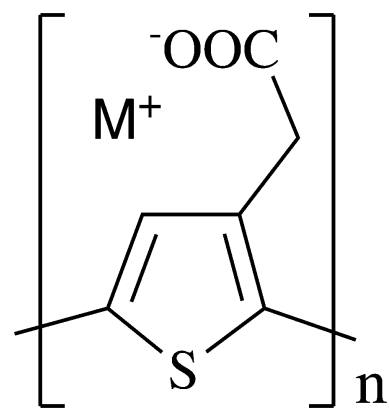

Fig. 1 Structural formula of the PTAA monomeric unit. 
They showed that the decrease of the counterion size (from tetrabutylammonium to ammonium) leads to a red-shift in the absorption spectrum of aqueous solutions of salts of poly(thiophen-3-ylpropionic acid). This effect was ascribed to the ability of larger counterions (e.g. tetrabutylammonium) to hinder polyion main chains from getting into contact with one another, thus preventing the aggregation of polyions while this is not the case for smaller counterions (e.g. tetramethylammonium). The strong dependence of the absorption and emission spectra on the length of alkyl chains in cationic surfactants added to aqueous solution of fully ionised regio-irregular PTAA confirmed the pronounced sensitivity of conjugated polyanions to their environment. ${ }^{31}$ In this case, observed changes in spectra were explained by the formation of mixed aggregates between polyion and surfactant molecules.

The goal of the present study is to address quantitatively the influence of the nature of counterions on the properties of the PTAA in solution. For this purpose, we have previously determined the fraction of free counterions, $f$, in aqueous solutions of PTAA lithium, sodium, and caesium salts using the combination of conductivity and transference number measurements. ${ }^{32}$ It turned out that $f$ does not depend on the nature of the alkali metal counterion, i.e. none of the mentioned alkali metal counterions seems to show specific interactions towards the poly(thiophen-3-ylacetate) polyion ( $\mathrm{PTA}^{-}$). In contrast, heats of dilution and heats of mixing strongly depend on the nature of the alkali metal counterion. ${ }^{33}$ This dependence, however, is most probably not directly related to the properties of PTAA but rather to the different affinities of the alkali counterions towards water. ${ }^{34}$ As a part of our latest study, ${ }^{33}$ heats of dilution of tetraalkylammonium salts of PTAA and heats of mixing of lithium PTAA salt (PTALi) with tetraalkylammonium chlorides were also determined. Measured heats of mixing PTALi solution with solutions of alkali metal chlorides on one side and with solutions of tetraalkylammonium chlorides on the other side were fundamentally different and the differences were ascribed to the existence of hydrophobic interactions between the PTAA hydrophobic backbone and the alkyl chains of tetraalkylammonium counterions. ${ }^{33}$ Considering that heats of mixing alone cannot provide information about the preferential binding of tetraalkylammonium over alkali counterions to $\mathrm{PTA}^{-}$, the current study investigates this competition further by measuring self-diffusion coefficients of counterions and polyions as well as by carrying out a thermodynamic analysis using both these new data and existing calorimetric data.

When association processes in solutions are studied, various NMR techniques can be used for the investigation of such phenomena. $^{35}$ For example, NMR quadrupole splitting has been used successfully for assessing the specific adsorption of lithium and sodium ions to dodecyl carboxylate and dodecyl sulphate bilayers $^{36}$ as well as for studying the specificity of binding of rubidium and caesium ions to dodecyl carboxylate in a liquid crystalline phase. ${ }^{37}$ However, for the system under study, i.e. for solutions of polyelectrolytes, the order parameter averages to zero and the NMR quadrupole splitting method cannot be used. In order to assess the physical origin of measured enthalpies of mixing, ${ }^{33}$ the process of mixing the polyion and the counterions was investigated by using another NMR method, namely the pulsed field gradient spin-echo NMR method (PFG SE NMR). By the use of PFG SE NMR it is possible to determine self-diffusion coefficients of the polyion and counterions that are valuable tools for the evaluation of counterion-polyelectrolyte interactions. ${ }^{38,39}$ Pulsed field gradient spin-echo NMR experiments were then performed at $298.1 \mathrm{~K}$ and, due to the lower sensitivity of NMR experiments, at somewhat higher (four-fold) concentrations than those used in the calorimetric measurements. The obtained self-diffusion coefficients were used to evaluate $f$ for every single counterion species involved in the mixing experiment. The experimental values were compared with $f$ derived from the solution of the Poisson-Boltzmann equation within the cell model of cylindrical symmetry. Finally, using the results of NMR experiments, the calorimetric data from mixing experiments were evaluated through a model thermodynamic analysis which enabled us to determine standard thermodynamic quantities for alkali metal counterions involved in mixing.

\section{Experimental part}

\subsection{Synthesis and sample preparation}

Synthesis. Detailed description of synthesis, characterisation and purification of PTAA is given in our previous papers, ${ }^{32,40}$ while only the most essential information is repeated here. As a starting material thiophen-3-ylacetic acid was used. This acid was esterified to give thiophen-3-ylacetic acid methyl ester and the latter one was converted to poly(methyl thiophen-3-ylacetate) by oxidative polymerisation. The polymerised product was fractionated on the basis of molar mass dependent solubility. The molar mass of each fraction was determined using size exclusion chromatography (SEC). ${ }^{32}$ The fraction with weightaverage molar mass $M_{\mathrm{w}}=13.4 \mathrm{~kg} \mathrm{~mol}^{-1}$ and number-average molar mass $M_{\mathrm{n}}=7.2 \mathrm{~kg} \mathrm{~mol}^{-1}$ (dispersity $D=M_{\mathrm{w}} / M_{\mathrm{n}}=1.86$ ) was converted to PTAA by alkaline hydrolysis with $\mathrm{NaOH}$ and used in further work.

Sample preparation for NMR experiments. Detailed description of sample preparation for NMR experiments is given in the $\mathrm{ESI} \dagger$ while only brief description is given here.

Lithium salt solution of PTAA was prepared by dissolving the

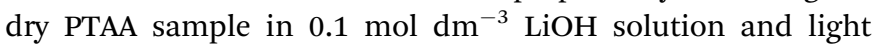
water $\left(\mathrm{H}_{2} \mathrm{O}\right)$ was removed afterwards by freeze-drying to obtain dry PTALi salt. The degree of neutralisation of PTAA with $\mathrm{LiOH}$ in the solution prepared for freeze-drying was around $97 \%$. This value was verified by the use of atomic absorption spectroscopy (concentration of $\mathrm{Li}^{+}$ions) and potentiometric titration of remaining non-neutralised carboxylic groups. Taking that PTAA is a representative of weak polyacids its degree of ionisation can be equalled with its degree of ionisation. The $\mathrm{pH}$ value of aqueous $\left(\mathrm{H}_{2} \mathrm{O}\right)$ PTAA solutions having a degree of neutralisation of $97 \%$ slightly depends on concentration and is around 8 . This holds true both for solutions without simple salt added and for solutions with low or moderate concentration of simple 
salt added. ${ }^{41}$ Complete neutralisation of PTAA was in our case avoided in order to prevent introducing surplus of $\mathrm{Li}^{+}$ions. When NMR experiments with these samples in deuterated water $\left(\mathrm{D}_{2} \mathrm{O}\right)$ are considered, some small departures from $\mathrm{pH}$ values measured in non-deuterated water can be expected. ${ }^{42}$ These differences, however, are too small to influence notably experimental results under given experimental conditions. The dried sample of PTALi was then dissolved in the corresponding amount of $\mathrm{D}_{2} \mathrm{O}$ to obtain $\mathrm{PE}$ solution with a concentration of 0.02 monomol $\mathrm{dm}^{-3}$. In order to prepare solutions with selected molar ratios $\left(r_{n}\right)$ between the added counterion and the PE's monomeric units, a corresponding volume of $0.2 \mathrm{~mol} \mathrm{dm}^{-3}$ simple salt solution was added to 0.02 monomol $\mathrm{dm}^{-3}$ solution of PTALi in $\mathrm{D}_{2} \mathrm{O}$. Here the molar ratio $r_{n}$ is defined as

$$
r_{\mathrm{n}}=\frac{c_{\mathrm{x}}}{c_{\mathrm{p}}}
$$

where $c_{\mathrm{x}}$ and $c_{\mathrm{p}}$ stand for the molar concentration of added simple salt and of PE (expressed in monomol $\mathrm{dm}^{-3}$ ) in the mixture, respectively. In mixing experiments simple salts such as lithium, sodium, caesium, tetramethylammonium $\left(\mathrm{TMA}^{+}\right)$, tetraethylammonium $\left(\mathrm{TEA}^{+}\right)$, tetrapropylammonium $\left(\mathrm{TPA}^{+}\right)$, and tetrabutylammonium $\left(\mathrm{TBA}^{+}\right)$chlorides were used.

The self-diffusion coefficients of $\mathrm{Li}^{+}$and of $\mathrm{PTA}^{-}$polyions (stemming from PTALi) as well as of the added counterion (from the simple salt added) were measured in prepared mixtures according to the procedure described in the following subsection.

\subsection{Measurements}

Self-diffusion coefficient measurement. Depending on the studied nucleus and on the sample characteristics, the requirements for PFG SE NMR experiments are different. Moreover, the accuracy of the obtained data also differs; further details are given in the ESI. $\dagger$

For ${ }^{7} \mathrm{Li}$ ions and ${ }^{1} \mathrm{H}\left(\mathrm{TMA}^{+}, \mathrm{TEA}^{+}, \mathrm{TPA}^{+}\right.$, and $\mathrm{TBA}^{+}$ions and $\mathrm{PTA}^{-}$polyions), a Bruker Avance DRX 500 NMR spectrometer operating at $499.76 \mathrm{MHz}$ for ${ }^{1} \mathrm{H}$ was used. Spectra were recorded using a ${ }^{1} \mathrm{H} / \mathrm{X}$ BBO broadband probe equipped with $z$-gradient coil producing $0.55 \mathrm{~T} \mathrm{~m}^{-1}$ at maximum. During these measurements the temperature was maintained at $298.1 \mathrm{~K}( \pm 0.1 \mathrm{~K})$.

For ${ }^{23} \mathrm{Na}$ and ${ }^{133} \mathrm{Cs}$ ions, higher gradients were required. Due to the fast relaxation of ${ }^{23} \mathrm{Na}$ nuclei magnetisation $\left(T_{1} \sim 20 \mathrm{~ms}\right)$, short and intense gradient pulses had to be used. In the case of ${ }^{133} \mathrm{Cs}$, the high gradient field was needed to compensate the low gyromagnetic ratio of this nucleus and to obtain sufficient signal attenuation (see eqn (2) and (3)). ${ }^{43}$ All these special demands, required by the properties of ${ }^{23} \mathrm{Na}$ and ${ }^{133} \mathrm{Cs}$ nuclei, were met by using a Bruker Avance III narrow bore Spectrometer operating at a magnetic field of $7.05 \mathrm{~T}$ equipped with a Diff30 gradient probe (producing $12 \mathrm{~T} \mathrm{~m}^{-1} \mathrm{~A}^{-1}$ at maximum) with a single (i.e. $z$ ) shielded gradient coil connected to a GREAT30 current amplifier capable of generating current pulses of $30 \mathrm{~A}$ amplitude.

All NMR experiments were performed in $\mathrm{D}_{2} \mathrm{O}$. A stimulated echo pulse sequence with longitudinal eddy current delay (BPP-LED, ${ }^{44}$ $T_{\mathrm{e}}=5 \mathrm{~ms}$ ) and bipolar gradient pulses of sine-shaped amplitude was used. During measurements the gradient of the magnetic field was increased in 16 increments (from $2 \%$ to $60 \%$ of maximal gradient strength for ${ }^{133} \mathrm{Cs}$ and from $2 \%$ to $90 \%$ for ${ }^{23} \mathrm{Na}$ ). Gradient pulse lengths were $\delta=2 \times 1 \mathrm{~ms}$ for ${ }^{133} \mathrm{Cs}$, and $\delta=2 \times 0.5 \mathrm{~ms}$ for ${ }^{23} \mathrm{Na}$ while the diffusion delay $\Delta$ was $100 \mathrm{~ms}$ for ${ }^{133} \mathrm{Cs}$ and $15 \mathrm{~ms}$ for ${ }^{23} \mathrm{Na}$.

The self-diffusion coefficient of counterions $D$ was calculated by fitting expression (2) to experimental points

$$
A(k)=A_{0} \cdot \mathrm{e}^{-k D}
$$

where $A(k)$ and $A_{0}$ represent the signal intensity in the presence and in the absence of the magnetic field gradient, respectively, and $k$ is defined as: ${ }^{44}$

$$
k=\delta^{2} g^{2} \gamma^{2}\left(\Delta-\frac{\delta}{3}-\frac{\tau}{2}\right)
$$

In the above equation, $\delta$ stands for the duration of gradient pulses, $g$ for the magnetic field gradient, $\gamma$ for the nuclear gyromagnetic ratio of the nucleus under study, $\Delta$ for the separation time between a pair of gradient pulses, and $\tau$ for the separation time between the second gradient pulse in the pair and the next radiofrequency pulse.

Regarding polyion self-diffusion coefficient $\left(D_{\mathrm{p}}\right)$ determination, a correction for sample dispersity was taken into account by using the equation ${ }^{45}$

$$
A(k)=A_{0} \cdot \mathrm{e}^{-k\langle D\rangle_{\mathrm{m}}+\frac{1}{2} k^{2}\left(\left\langle D^{2}\right\rangle_{\mathrm{m}}-\langle D\rangle_{\mathrm{m}}^{2}\right)}
$$

where $\left\langle D^{2}\right\rangle_{\mathrm{m}}$ and $\langle D\rangle_{\mathrm{m}}$ represent the mass average of the squared polyion self-diffusion coefficient and the mass average of the polyion self-diffusion coefficient, respectively. In dilute solutions, it is possible to use a scaling approach ${ }^{46}$

$$
D(M) \approx M^{-\alpha}
$$

which takes into account the dependence of the self-diffusion coefficient on the molecular mass. In eqn (5), $D(M)$ represents the molar-mass dependent self-diffusion coefficient of the polyion, $M$ the molar mass of observed species, and $\alpha$ is a positive parameter related to the average conformation of the polymer. In the case of a random coil, the value of $\alpha$ is 0.6 while for the non-draining ellipsoid the value is $0.33 .^{46}$

Assuming a log-normal polymer molar mass distribution, $\left\langle D^{2}\right\rangle_{\mathrm{m}}$ and $\langle D\rangle_{\mathrm{m}}$ can be computed using eqn (5) since all the moments of the distribution are known. Eqn (4) is then rewritten as (see the ESI $\dagger$ for derivation):

$$
A(k)=A_{0} \cdot \mathrm{e}^{-k\langle D\rangle_{\mathrm{m}}+\frac{1}{2} k^{2}\langle D\rangle_{\mathrm{m}}^{2}}\left(D^{\alpha^{2}}-1\right)
$$

During fitting, the value of dispersity $Ð$ was kept constant at 1.86 (the value determined by SEC), while the mass-averaged polyion self-diffusion coefficient $\left(D_{\mathrm{p}} \equiv\langle D\rangle_{\mathrm{m}}\right)$ and $\alpha$ were used as adjustable parameters.

The measured self-diffusion coefficient of the counterions was used to evaluate the fraction of free counterions, $f$, a quantity that has in the case of PE solution a similar value as the osmotic coefficient or the activity coefficient of counterions. ${ }^{47}$ In PE solutions, $f$ can be obtained from the ratio between the measured self-diffusion coefficient of the counterion 
$(D)$ and the self-diffusion coefficient of the given counterion in the limit of infinite dilution $\left(D^{\circ}\right)$. In order to eliminate the contribution of counterions bound to polyions to diffusion processes, in our case the self-diffusion of the polyion $\left(D_{\mathrm{p}}\right)$ was also taken into account:

$$
f=\frac{D-D_{\mathrm{p}}}{D^{\circ}-D_{\mathrm{p}}}
$$

Ultraviolet-visible absorption spectra measurements. UV/vis absorption spectra were recorded at $298.1 \mathrm{~K}$ in a quartz cuvette with the optical path of $0.025 \mathrm{~cm}$ using a Varian Cary $100 \mathrm{UV} / \mathrm{vis}$ spectrophotometer. During these experiments aqueous solution of PTALi $\left(c_{\mathrm{p}}=0.005\right.$ monomol $\left.\mathrm{dm}^{-3}\right)$ was mixed with such a volume of simple salt solution $\left(0.15 \mathrm{~mol} \mathrm{dm}^{-3}\right)$ that the desired value of $r_{n}$ was achieved. The cuvette was filled with the solution and the absorption spectrum in the interval from $800 \mathrm{~nm}$ to $200 \mathrm{~nm}$ was measured.

\section{Theoretical part}

\subsection{Cylindrical cell model}

In order to estimate $f$ in the salt-free PE solution as well as in the PE solution with the simple salt added, the PoissonBoltzmann equation in cylindrical symmetry ${ }^{47-49}$ was solved, and its solution was used in the theoretical prediction of $f$. This approach takes into account only electrostatic interactions and ignores possible specific interactions stemming from the hydrophobic effect. Taking that this theory is frequently used in the scientific literature, the calculation procedure is omitted here and is given in the ESI $\dagger$ instead.

\subsection{Thermodynamic analysis of cation-polyion association}

Calorimetric data obtained in our previous study ${ }^{33}$ cannot provide per se a molecular insight into the processes occurring when PE solution is mixed with simple salt solution. In order to get such a picture from calorimetric data, a new model, based on our previous work, ${ }^{50,51}$ was developed. In this new model, besides calorimetric data, measured fractions of free counterions are used as relevant input parameters.

It is assumed that each monomeric unit of the polymer can be represented as one independent binding site. This binding site can be either unoccupied (this is a model representation of the $-\mathrm{COO}^{-}$group where no counterion is present in its immediate vicinity) or occupied (a counterion is bound to the $-\mathrm{COO}^{-}$group thus screening its charge effectively). In the pure PE solution without added simple salt (in our case this corresponds to PTALi solution) there exists an equilibrium between unoccupied and with lithium counterions occupied binding sites. When simple salt is added, the added counterions compete with the already present lithium counterions for unoccupied binding sites. This new equilibrium in solution can be written down as:

$$
\text { PTALi } \underset{-\mathrm{Li}^{+}}{\stackrel{+\mathrm{Li}^{+}}{\leftrightarrows}} \text { PTA }^{-} \underset{-\mathrm{M}^{+}}{\stackrel{+\mathrm{M}^{+}}{\leftrightarrows}} \text { PTAM }
$$

where PTA ${ }^{-}$, PTALi, and PTAM represent an unoccupied binding site, a binding site occupied by the lithium ion, and the one occupied by the added counterion, respectively. There also exists a competition for occupied binding sites but this competition is indirect via unoccupied sites. The enthalpy change accompanying the addition of simple salt at a given temperature, $\Delta_{\text {add }} H_{x}(T)$, can be expressed as ${ }^{52,53}$

$$
\Delta_{\mathrm{add}} H_{x}(T)=\sum_{i} \Delta_{\mathrm{b}} H_{i}(T)\left(\frac{\partial n_{i}}{\partial n_{x}}\right)_{p, T, n_{1}}
$$

where $\Delta_{\mathrm{b}} H_{i}(T)$ represents the enthalpy of binding of the $i$-type counterion to an unoccupied binding site and $\left(\partial n_{i} / \partial n_{x}\right)_{p, T, n_{1}}$ denotes the partial derivative of the amount of binding sites $n_{i}$ occupied by counterion $i$ with respect to the total amount of added simple salt $n_{x}$ at given $p, T$ and constant amount of solvent $\left(n_{1}\right)$. When eqn (8) is applied to above described counterionpolyion interactions and if it is assumed that $\Delta_{\mathrm{b}} H_{i}(T)$ is not dependent on concentration, eqn (8) transforms into

$$
\begin{aligned}
\Delta_{\mathrm{add}} H_{x}(T)= & \Delta_{\mathrm{b}} H_{\mathrm{Li}}^{\ominus}(T)\left(\frac{\partial[\mathrm{PTALi}]}{\partial c_{\mathrm{x}}}\right)_{p, T, n_{1}} \\
& +\Delta_{\mathrm{b}} H_{\mathrm{M}}^{\ominus}(T)\left(\frac{\partial[\mathrm{PTAM}]}{\partial c_{\mathrm{x}}}\right)_{p, T, n_{1}}
\end{aligned}
$$

where $\Delta_{\mathrm{b}} H_{\mathrm{M}}^{\ominus}(T)$ stands for the standard molar enthalpy of binding of counterion $\mathbf{M}$ and square brackets denote the equilibrium concentration of given species in solution. The latter concentrations can be calculated from the total concentration of added salt $\left(c_{\mathrm{x}}\right)$, monomeric unit concentration $\left(c_{\mathrm{p}}\right)$, and equilibrium constant for binding of the lithium ion (eqn (10)) and of the added counterion (eqn (11)), respectively.

$$
\begin{aligned}
K_{\mathrm{Li}} & =\frac{[\mathrm{PTALi}]}{\left[\mathrm{Li}^{+}\right]\left[\mathrm{PTA}^{-}\right]} \\
K_{\mathrm{M}} & =\frac{\left[\mathrm{PTAM}^{2}\right]}{\left[\mathrm{M}^{+}\right]\left[\mathrm{PTA}^{-}\right]}
\end{aligned}
$$

The temperature dependence of standard molar Gibbs energies, $\Delta_{\mathrm{b}} G_{i}^{\ominus}(T)$, and enthalpies of binding can be expressed by the Gibbs-Helmholtz relation (eqn (12)) and by the Kirchhoff law (eqn (13)).

$$
\begin{gathered}
\Delta_{\mathrm{b}} G_{i}^{\ominus}(T)=T\left\{\frac{\Delta_{\mathrm{b}} G_{i}^{\ominus}\left(T_{0}\right)}{T_{0}}+\Delta_{\mathrm{b}} H_{i}^{\ominus}\left(T_{0}\right)\left[\frac{1}{T}-\frac{1}{T_{0}}\right]\right. \\
\left.+\Delta_{\mathrm{b}} C_{i, p}^{\ominus}\left[1-\frac{T_{0}}{T}-\ln \left(\frac{T}{T_{0}}\right)\right]\right\} \\
\Delta_{\mathrm{b}} H_{i}^{\ominus}(T)=\Delta_{\mathrm{b}} H_{i}^{\ominus}\left(T_{0}\right)+\Delta_{\mathrm{b}} C_{i, p}^{\ominus}\left(T-T_{0}\right)
\end{gathered}
$$

The use of the above written relations allows us to describe the binding of each type of counterion with a single set of adjustable parameters $K_{i}, \Delta_{\mathrm{b}} H_{i}^{\ominus}\left(T_{0}\right)$, and the difference in the molar heat capacities of products and reactants $\left(\Delta_{\mathrm{b}} C_{i, p}^{\ominus}\right)$, respectively (keep in mind that $\Delta_{\mathrm{b}} G_{i}^{\ominus}(T)=-R T \ln \left(K_{i}\right)$ at arbitrary $T$ ). In order to minimize the number of adjustable parameters, the equilibrium constants $K_{i}$ can be calculated from the measured fractions of free counterions using the approximation that the activity coefficient of counterions is equal to $f$ and that activity 


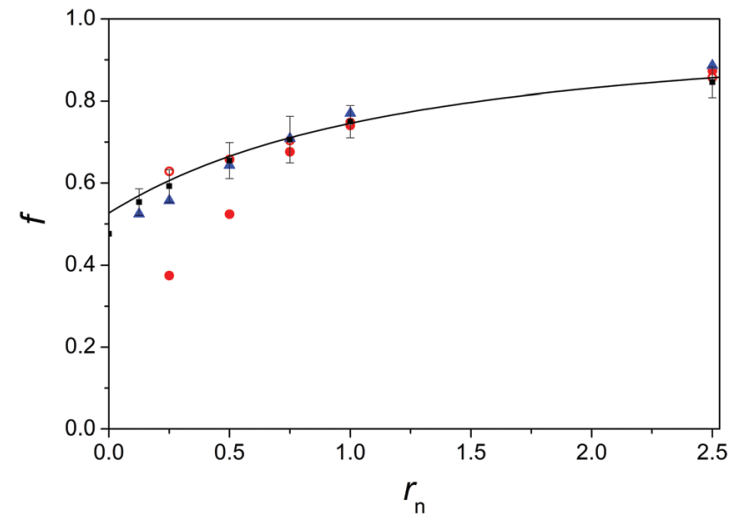

Fig. 2 Fraction of free lithium ( $\mathbf{\square})$, sodium ( - from attenuation of signal intensity and $\bigcirc$ - from attenuation of peak area) and caesium ( $\mathbf{\Delta}$ ) counterions as a function of the ratio $r_{n}$ between the concentration of simple salt and the monomolar concentration of PTALi. The data were obtained upon mixing 0.020 monomol dm $\mathrm{m}^{-3}$ aqueous solution of PTALi with $0.2 \mathrm{~mol} \mathrm{dm}^{-3}$ $\mathrm{LiCl}, \mathrm{NaCl}$ and $\mathrm{CsCl}$ solutions at $298.15 \mathrm{~K}$, respectively. Error-bars represent the estimated error in $f$ while the continuous line denotes prediction based on the cylindrical cell model. See the text for further details.

coefficients of $-\mathrm{COO}^{-}$and $-\mathrm{COO}^{-} \mathrm{M}^{+}$species are equal to 1 . Although the first approximation is believed to hold rather well, ${ }^{47}$ the validity of the last two is more questionable. Therefore, $K_{i}$ are not the true thermodynamic constants but the apparent ones.

Since previous ${ }^{32}$ and current experiments (see Fig. 2 in Section 4.1) show that the fractions of free lithium, sodium, and caesium counterions in salt-free PTAA solutions are equal within the experimental error, it is assumed that $K_{\mathrm{Li}}=K_{\mathrm{Na}}=K_{\mathrm{Cs}}$. The binding constant $K_{\mathrm{Li}}$ was estimated from the analytical concentration $\left(c_{\mathrm{Li}}\right)$ and the fraction of free lithium counterions $\left(f_{\mathrm{Li}}\right)$ at $r_{n}=0\left(\left[\mathrm{Li}^{+}\right]=\left[\mathrm{PTA}^{-}\right]=c_{\mathrm{Li}} f_{\mathrm{Li}}\right.$ and $\left.[\mathrm{PTALi}]=c_{\mathrm{Li}} \cdot\left(1-f_{\mathrm{Li}}\right)\right)$. The values of remaining adjustable parameters $\left(\Delta_{\mathrm{b}} H_{i}^{\ominus}\left(T_{0}\right)\right.$ and $\Delta_{\mathrm{b}} C_{i, p}^{\ominus}$ ) were finally determined by the global fitting of the model function to the experimental calorimetric isothermal titration curves, measured at various temperatures and for titrations with different alkali metal salts ( $\mathrm{LiCl}, \mathrm{NaCl}, \mathrm{CsCl})$.

\section{Results and discussion}

\subsection{Fractions of free counterions}

In Fig. 2 and 3 fractions of free alkali metal (Fig. 2) and tetraalkylammonium (Fig. 3) counterions in PTALi solutions containing various amounts of simple salts added are shown. The presented experimental points were obtained during the mixing of 0.020 monomol $\mathrm{dm}^{-3}$ aqueous solution of PTALi with $0.2 \mathrm{~mol} \mathrm{dm}^{-3}$ solutions of alkali metal chlorides ( $\mathrm{LiCl}, \mathrm{NaCl}$, and $\mathrm{CsCl}$ ), and tetraalkylammonium chlorides (TMACl, TEACl, TPACl, and TBACl), respectively. Measured values are shown as a function of $r_{\mathrm{n}}$, representing a ratio between the concentration of simple salt added and the monomolar concentration of $\mathrm{PTA}^{-}$.

Alkali counterion addition. There is a faint indication that other added counterions might displace bound $\mathrm{Li}^{+}$ions to an extent which slightly exceeds the statistical one (see Fig. S2 in the ESI $\dagger$ )

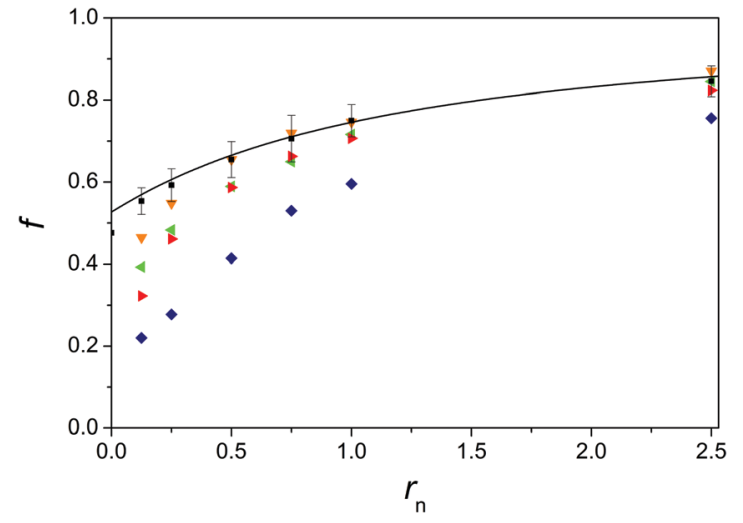

Fig. 3 The same as in Fig. 2 but for the addition of tetraalkylammonium chlorides (TMACl, TEACl, TPACl, and TBACl) to PTALi. Symbols: $\mathrm{Li}^{+}(\mathbf{\square})$, $\operatorname{TMA}^{+}(\nabla), \operatorname{TEA}^{+}\left(\langle), \operatorname{TPA}^{+}(\gg)\right.$, and $\operatorname{TBA}^{+}()$.

but this observation might also be easily a consequence of the experimental error.

Therefore, $f_{\mathrm{Li}}$ values in Fig. 2 and 3 are shown as an average of values obtained for the addition of all simple salts whereas error bars show the largest deviation of measured values from the average value of $f_{\mathrm{Li}}$.

As one can see, $f$ increases in all cases with increasing $r_{\mathrm{n}}$. Such a trend is also predicted by electrostatic theories: as an example of such calculations the result of the cylindrical cell model ( $a=0.4 \mathrm{~nm}, b=0.38 \mathrm{~nm}$ ) is depicted by continuous black lines in both figures. Since counterions are considered to be pointlike in these calculations, this line holds for all monovalent salts of PTAA. It is possible to mimic the effect of finite radii of counterions by increasing the radius $a$ of the polyion in these calculations ( $a$ can also be regarded as a distance of the closest approach of the centre of counterions to the axis of the polyion). Such calculations would show that increasing the radius of counterions leads to slightly larger values of $f$. As can be seen in Fig. 2, theoretical and measured values agree very well for the case of lithium and caesium counterions whereas for sodium ones the agreement is apparently good only at large enough $r_{n}$ values. The reason for sodium deviation at $r_{n}=0.25$ and 0.5 is most probably an experimental error which can be attributed to the low signal-to-noise ratio stemming from the short relaxation time of the ${ }^{23} \mathrm{Na}$ nucleus (see the ESI $\dagger$ ).

Fig. 2 shows that sodium counterions bind to $\mathrm{PTA}^{-}$to a similar extent as other alkali ions for large values of $r_{n}$ and the values of $f$ measured in solutions of alkali metal salts of PTAA suggest ${ }^{32}$ that this is also valid at low $r_{n}$ values.

In the absence of more accurate experimental results, it will be assumed that in the case of alkali metal ions no preferential binding of counterions to $\mathrm{PTA}^{-}$exists. The exchange of different alkali metal counterions bound to $\mathrm{PTA}^{-}$therefore seems to be of statistical nature. In addition, regarding the concordance of measured $f$ of alkali metal counterions with the ones calculated from the theory it is possible to say that electrostatic interactions satisfactorily explain the binding of alkali metal counterions to the polyanion. Yet, the observation that all the studied alkali metal counterions are bound to the $\mathrm{PTA}^{-}$polyion 
approximately to the same extent tells nothing about details how ${ }^{34,54,55}$ these counterions are bound. The used PFG SE NMR method cannot give such a kind of information and other methods should be used for this purpose.

From $f$ it is possible to calculate the fraction of charged groups on the polyion counterbalanced by bound counterions (also known as the degree of condensation or the fraction of occupied binding sites, depending on the model used), $\theta$. This quantity is for some purposes a better indicator of processes occurring on the polyion than $f$. If one calculates $\theta$ from theoretically computed $f$ as

$$
\theta=(1-f) \cdot\left(1+r_{\mathrm{n}}\right)
$$

we see (Fig. S3 in the ESI $\dagger$ ) that $\theta$ has rather a constant value of about 0.5 for all values of $r_{\mathrm{n}}$. This means that the addition of new alkali metal counterions does not change $\theta$ but merely leads to the exchange of bound counterions with the added ones. These results confirm the assumption made in our previous paper $^{33}$ that differences in heats of mixing are not a consequence of different fractions of bound alkali metal counterions, but rather of different molar enthalpies of the hydration of counterions already present in solution and of the added counterion.

Tetraalkylammonium counterion addition. In Fig. 3, the dependence of the fraction of free tetraalkylammonium counterions on $r_{n}$ is compared with the prediction based on the cylindrical cell model (continuous black line; please recall that this prediction largely coincides with values of $f$ measured for the addition of alkali metal chloride solutions). We can see that, similar to alkali metal counterions, the values of $f$ of tetraalkylammonium counterions also increase with $r_{n}$. In contrast to alkali metal counterions, tetraalkylammonium counterions show a distinctive trend for $f$ with the size of the counterion (the greater the counterion, the lower the fraction of free counterions) and this trend is opposite to the one predicted by electrostatics.

The highest $f$ is exhibited by the smallest counterion, i.e. the tetramethylammonium $\left(\mathrm{TMA}^{+}\right)$ion. Measured $f_{\mathrm{TMA}}$ is almost identical to those predicted by electrostatics and measured for alkali metal counterions. The values of $f_{\text {TMA }}$ deviate considerably (beyond experimental error) from those observed for alkali metal counterions only at $r_{n}=0.125$ and $r_{n}=0.25$. However, this deviation is (at least partially) an artefact of measurements, as for ${ }^{23} \mathrm{Na}$, though for a different reason. For $\mathrm{TMA}^{+}$, it arises from the overlap of the ${ }^{1} \mathrm{H}$ NMR signal of TMA ${ }^{+}$and $\mathrm{PTA}^{-}$peaks (see the ESI $\dagger$ ). For larger values of $r_{n}$, the ratio of the intensity of $\mathrm{PTA}^{-}$to $\mathrm{TMA}^{+}$peaks is much smaller and consequently the error diminishes. Taking this factor into account, it may be claimed that the values of $f_{\text {TMA }}$ do not deviate considerably from fractions of free alkali metal counterions. This observation is also in line with some earlier findings. ${ }^{56}$

In the case of tetraalkylammonium counterions with longer alkyl chains $\left(\mathrm{TEA}^{+}, \mathrm{TPA}^{+}\right.$, and $\mathrm{TBA}^{+}$) there exist counterion peaks in the ${ }^{1} \mathrm{H}$ NMR spectrum which are well separated from the polyion peaks and difficulties related to the peak overlap vanish. The effect of diminishing $f$ with the increased length of alkyl chains is especially pronounced at low values of $r_{n}$. To be more specific, at $r_{n}=0.125$ approximately $60 \%$ of TEA $^{+}, 70 \%$ of $\mathrm{TPA}^{+}$, and $80 \%$ of $\mathrm{TBA}^{+}$counterions are bound to the polyion. Obviously, in the case of counterions with longer alkyl chains, the polyion-counterion association is driven by some additional attractive interactions. These interactions, provoked probably by the presence of hydrophobic thiophene rings, should at least partially contribute to the measured enthalpies of mixing and thus to the different trends in the dependence of enthalpies of mixing on hydration enthalpies of tetraalkylammonium counterions. ${ }^{33}$ With increasing $r_{n}$ differences in $f$ diminish.

The plot of the total fraction of occupied sites, $\theta_{\text {tot }}$, and the fraction of sites occupied by lithium counterions, $\theta_{\mathrm{Li}}$, versus $r_{n}$ (Fig. 4) shows an even more interesting picture. The values of $\theta_{\text {tot }}$ are here calculated from the experimentally determined $f_{\mathrm{Li}}$ and fractions of free added tetraalkylammonium counterions, $f_{\text {TAA }}$, as

$$
\theta_{\mathrm{tot}}=\left(1-f_{\mathrm{Li}}\right)+\left(1-f_{\mathrm{TAA}}\right) \cdot r_{n}
$$

Whereas for moderate $\left(r_{n}<1\right)$ additions of TMACl, TEACl, and TPACl, the total fraction of occupied sites $\theta_{\text {tot }}$ is just slightly above 0.5 and due to the experimental error no preferential binding among $\mathrm{TMA}^{+}, \mathrm{TEA}^{+}$or $\mathrm{TPA}^{+}$counterions can be established, and at higher values of $r_{n}$ one can find a distinctive increase of $\theta_{\text {tot }}$ with the increase of alkyl chain length. At $r_{n}=2.5$ for $\mathrm{TMA}^{+} \theta_{\text {tot }}=0.47$, for $\mathrm{TEA}^{+} 0.55$, for $\mathrm{TPA}^{+} 0.60$, and for $\mathrm{TBA}^{+} 0.77$. In spite of increased binding of counterions with longer alkyl chains $\theta_{\mathrm{Li}}$ remains unaffected by the kind of added counterion.

Since the fraction of bound counterions (at $r_{n}=2.5, \theta_{\text {tot }}$ is about $50 \%$ greater for the addition of $\mathrm{TBA}^{+}$than for $\mathrm{TMA}^{+}$) increases with the length of the alkyl chain in counterions, one

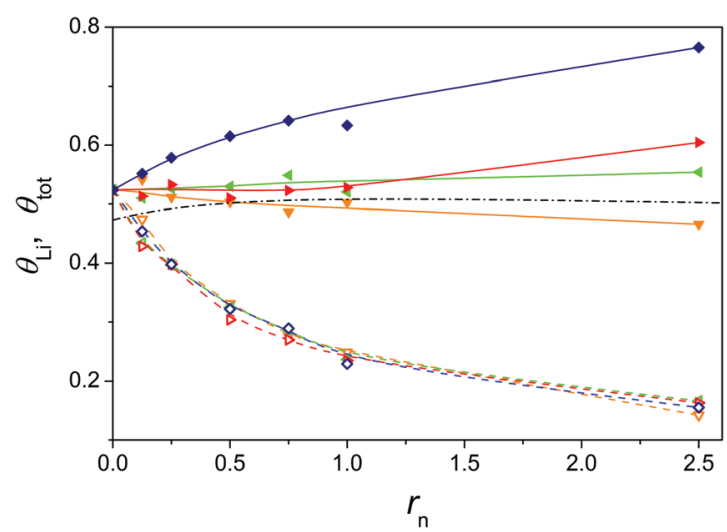

Fig. 4 Total fraction $\theta_{\text {tot }}$ (solid symbols) and fraction by lithium counterion $\theta_{\mathrm{Li}}$ (open symbols) occupied binding sites on $\mathrm{PTA}^{-}$upon addition of tetraalkylammonium chlorides. Symbols: addition of (TMACl $(\nabla, \nabla), \mathrm{TEACl}$ $(\triangleleft, \triangleleft), \operatorname{TPACl}(\triangleright, \triangleright)$, and $\operatorname{TBACl}(\diamond, \diamond))$. Data were obtained upon mixing 0.020 monomol dm ${ }^{-3}$ PTALi aqueous solution with $0.2 \mathrm{~mol} \mathrm{dm}^{-3}$ tetraalkylammonium chloride solutions at $298.1 \mathrm{~K}$. Continuous lines $\left(\theta_{\text {tot }}\right)$ and dashed lines $\left(\theta_{\mathrm{Li}}\right)$ through the symbols are intended to guide the eye. The black dash-dotted line represents $\theta_{\text {tot }}$ calculated from the cylindrical cell model (only coulombic interactions are taken into account). 
might expect that the conformation of the $\mathrm{PTA}^{-}$polyion is dependent on the kind of counterion.

\subsection{Conformation of the polyion}

A transition of the conjugated polyion from an extended conformation to a more compact one may be evidenced, among others, from the change in the polyion self-diffusion coefficient $\left(D_{\mathrm{p}}\right)$ and from the position of the absorption maximum in its $\mathrm{UV} /$ vis spectrum.

In the present case, the addition of any simple salt to PTALi solution leads to the increase of $D_{\mathrm{p}}$ as $r_{n}$ increases (Fig. 5). Regarding differences among results obtained for various simple salts one has to say that the relative error in measured self-diffusion coefficients can exceed $5 \%$ (the estimated uncertainty is shown in Fig. 5 by error bars) (see the ESI $\dagger$ ). Despite the experimental uncertainty, it seems that two counterions $\left(\mathrm{TPA}^{+}\right.$ and $\mathrm{TBA}^{+}$) have a more pronounced effect on $D_{\mathrm{p}}$ than the other ones. This holds true especially for $\mathrm{TBA}^{+}$where distinctively larger values of $D_{\mathrm{p}}$ are observed at all $r_{n}$ values. For TPA ${ }^{+}$, noticeably different values are present only at high $r_{n}$. Regarding the addition of salts, presented in Fig. 5, the smallest increase of $D_{\mathrm{p}}$ is observed for the addition of $\mathrm{LiCl}$, while the remaining two studied simple salts (i.e. $\mathrm{NaCl}$ and $\mathrm{CsCl}$; not shown in Fig. 5) give similar to or slightly smaller effects than the addition of LiCl.

The observed general trend of increasing $D_{\mathrm{p}}$ with increasing $r_{n}$ can be ascribed to the screening of electrostatic forces in solution. In principle, electrostatic forces could stem either from inter- or intramolecular interactions. It is known that screening of intermolecular interactions is the most probable reason for the increase of 'slow' diffusion coefficient ${ }^{57}$ observed in dynamic light scattering (DLS) experiments during the addition of simple salt to PE solution. On the other hand, the 'slow' diffusion coefficient cannot be equated using the selfdiffusion process ${ }^{58}$ and the increase of $D_{\mathrm{p}}$ therefore cannot be unambiguously ascribed to screening of intermolecular interactions. Consequently, the trend observed in Fig. 5 may be at least partially a result of the screening of intramolecular

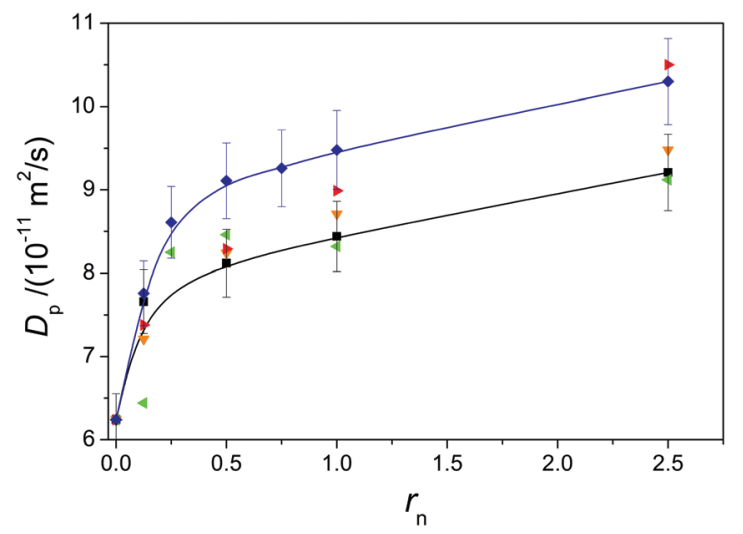

Fig. 5 Dependence of the self-diffusion coefficient of the polyion $\left(D_{p}\right)$ on ratio $r_{n}$ obtained during mixing 0.020 monomol $\mathrm{dm}^{-3}$ PTALi aqueous solution with $0.2 \mathrm{~mol} \mathrm{dm}^{-3} \mathrm{LiCl}, \mathrm{TMACl}, \mathrm{TEACl}, \mathrm{TPACl}$, and TBACl at $298.15 \mathrm{~K}$, respectively. Legend as in Fig. 3. Lines are used to guide the eye. electrostatic forces by added simple salt, which provokes transition to more constricted conformation of the polyion. Using the Stokes-Einstein relation, one can estimate the hydrodynamic radius of the PTALi polyion $\left(D_{\mathrm{p}}=9.1 \times 10^{-11} \mathrm{~m}^{2} \mathrm{~s}^{-1}\right.$; see Fig. 5) at $r_{n}=2.5$ to lie around $2.7 \mathrm{~nm}$, which is a reasonable estimate for our sample.

The effect of screening of intramolecular electrostatic forces observed through the dependence of $D_{\mathrm{p}}$ on $r_{n}$ can be verified in Fig. 6 , where $\lambda_{\max }$ is the position of the absorption maximum in the UV/vis spectrum of $5 \times 10^{-3}$ monomol $\mathrm{dm}^{-3}$ solution of PTALi, as a function of $r_{n}$ and the nature of added salt is reported. When solutions of alkali metal chlorides are added to PTALi solution, the position of the absorption maximum does not change notably (these data are omitted in Fig. 6). $\lambda_{\max }$ does not change either upon TMACl addition, similar to what was observed for $f$. In contrast, upon addition of TEACl to PTALi, a blue shift of up to $5 \mathrm{~nm}$ is observed. With TPACl, the blue shift is slightly more pronounced $(7 \mathrm{~nm})$ while the largest shift is observed during titration with TBACl where $\lambda_{\max }$ shifts for about $20 \mathrm{~nm}$ towards lower wavelengths. As for $D_{\mathrm{p}}$, the main change in $\lambda_{\text {max }}$ takes place at low $r_{n}$ values $\left(r_{n}<0.5\right)$.

The blue shift in the spectra of conjugated PEs can be explained by two phenomena. The first one is a dissolution of $\pi$-aggregates ${ }^{28}$ and the second one is the collapse of the polymer chain that leads to a shorter effective conjugation length. ${ }^{59}$ In the classical study of McCullough et al. the blue shift observed during the addition of different counterions to aqueous solution of regio-regular poly(thiophen-3-ylpropionic acid) was explained by the decomposition of $\pi$-aggregates. ${ }^{28}$ On the other side, in our study regio-irregular PTAA was used and the blue shift can hardly be ascribed to the disaggregation of $\pi$-aggregates due to the following reasons: (i) blue shifts observed in the study of McCullough et al. ${ }^{28}$ are much more pronounced than those found here (in the case of TBACl $130 \mathrm{~nm}$ vs. $20 \mathrm{~nm}$ observed in this study); (ii) the formation of $\pi$-aggregates is far less likely to occur in the case of regioirregular conjugated polymers than in the case of regio-regular ones; ${ }^{60}$ (iii) the disappearance of $\pi$-aggregates would very likely

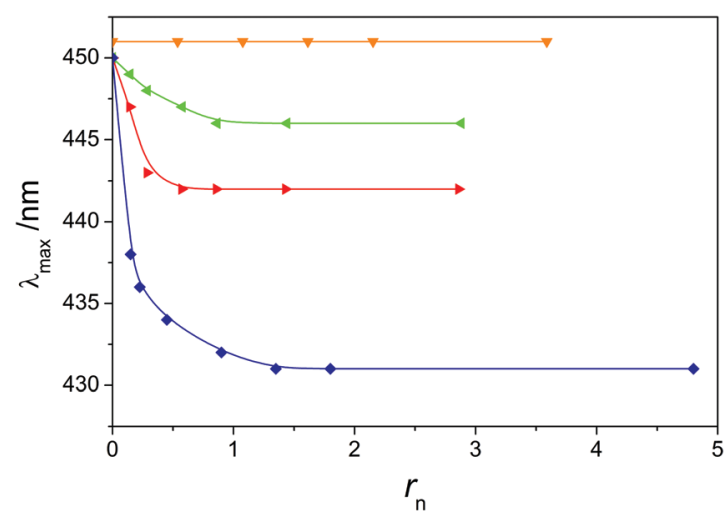

Fig. 6 Position of the maximum of the UV/vis absorption spectra as a function of $r_{n}$ for the titration of 0.005 monomol dm ${ }^{-3}$ PTALi aqueous solution with different tetraalkylammonium chlorides. Legend as in Fig. 3. Lines are used to guide the eye. 
influence the shape of the absorption spectrum; ${ }^{60}$ (iv) it was shown by X-ray scattering that no reversible aggregates were formed in aqueous solutions of sodium salt of the regio-irregular PTAA sample having a similar molar mass as the sample used in our study. ${ }^{30}$ Considering also the above mentioned arguments, we ascribe the blue shift observed during the addition of tetraalkylammonium counterions with longer alkyl chains to the transition of the polyion to a more compact conformation.

The most pronounced blue shift, provoked by the addition of $\mathrm{TBA}^{+}$counterions, can be explained by a stronger/more extensive counterion binding (higher $\theta_{\text {tot }}$, see Fig. 4) which leads to a more effective screening of electrostatic forces on the polyion. The correlation between $\theta_{\text {tot }}$ and the blue shift holds quite well also for other tetraalkylammonium counterions: they both increase by increasing the length of alkyl chains in these counterions. On the other side, $D_{\mathrm{p}}$ increases irrespective of the kind of simple salt added while $\lambda_{\max }$ remains unchanged only in the case of the addition of alkali metal salts and TMACl. This might be a consequence of the transition of the polyion to a more compact conformation which does not affect notably an effective conjugation length. Namely, certain theoretical studies $^{61,62}$ have shown that the moderate loss of coplanarity does not change significantly the conjugation length in polythiophenes.

\subsection{Thermodynamic analysis}

Fractions of free counterions, obtained in the current study, are valuable information that supplements the enthalpies of mixing, $\Delta_{\text {mix }} H$, measured in our previous study. ${ }^{33}$ The study showed that $\Delta_{\text {mix }} H$ measured during the mixing of PTALi with alkali and tetraalkylammonium chlorides has distinctly a different dependence on the enthalpy of hydration of the salt cation, $\Delta_{\text {hyd }} H$. This different dependence was ascribed to the dissimilar nature of both groups of cations (i.e. hydrophilic and hydrophobic) and to leaving/entering of water molecules from/to solvent sheaths of solutes being dependent on the nature of counterions. Due to uncertainties related to possible preferential binding of certain kinds of counterions more detailed analysis of calorimetric data at that moment would be pure speculation and was therefore omitted. By having determined $f$ for every kind of counterion under similar conditions at which $\Delta_{\text {mix }} H$ was measured it is then possible to keep one step forward and to make use of thermodynamic analysis described in Section 3.2 of this paper. The reader should be aware that the calorimetric data, although being the same, are shown in ref. 33 as cumulative heats of mixing per mole of monomeric units (in order to compare the figures with theoretical calculations of Boyd et al. ${ }^{63}$ ) while in the present analysis differential heats of mixing, $\Delta_{\mathrm{dmix}} H$, expressed per mole of added simple salt are used.

In our thermodynamic analysis, the standard Gibbs energy of binding, $\Delta_{\mathrm{b}} G_{i}^{\ominus}(T)$, of a given counterion $i$ to the $\mathrm{PTA}^{-}$ polyion was directly calculated from the apparent equilibrium constant $K_{i}$ for binding of counterion $i$. Following the results of one of our previous studies ${ }^{32}$ and the present NMR data (see Fig. 2), $K_{i}$ values (and thus $\Delta_{\mathrm{b}} G_{i}^{\ominus}(T)$ ) were set equal for all three added alkali counterions. A single optimization procedure was

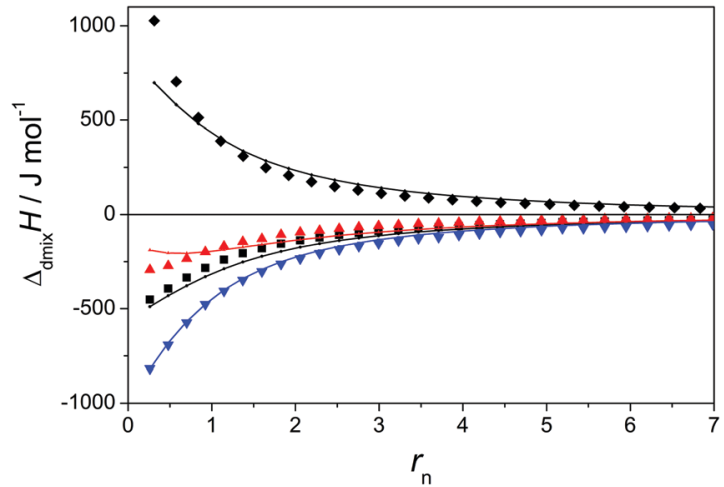

Fig. 7 Comparison of experimental ${ }^{33}$ (symbols) differential enthalpies of mixing, $\Delta_{\text {dmix }} H$, and results of global fitting (lines) for the titration of 0.005 monomol dm ${ }^{-3}$ PTALi with $0.15 \mathrm{~mol} \mathrm{dm}^{-3} \mathrm{NaCl}(278.15 \mathrm{~K}-\nabla, 298.15 \mathrm{~K}-$ $\mathbf{\square}$, and $318.15 \mathrm{~K}-\boldsymbol{\Delta})$ and for reverse titration $\left(0.005\right.$ monomol $\mathrm{dm}^{-3}$ PTANa with $\left.0.15 \mathrm{~mol} \mathrm{dm}^{-3} \mathrm{LiCl}\right)$ at $298.15 \mathrm{~K}(\bullet)$.

then used to determine $\Delta_{\mathrm{b}} H_{i}^{\ominus}(T), \Delta_{\mathrm{b}} S_{i}^{\ominus}(T)$, and $\Delta_{\mathrm{b}} C_{i, p}^{\ominus}(T)$ from calorimetric titration curves at all given temperatures $(278.15 \mathrm{~K}$, $298.15 \mathrm{~K}$, and $318.15 \mathrm{~K})$ for all alkali counterions simultaneously. Due to the reliability of data, values of $K_{i}$ were determined from $K_{\mathrm{Li}}$ whereas the value of $K_{\mathrm{Li}}$ was calculated from $f_{\mathrm{Li}}$ at $r_{n}=0$. The agreement between experimental and model values of mixing of PTALi with $\mathrm{NaCl}$ (Fig. 7) and of mixing of PTALi with CsCl (Fig. 8) is reasonably good. The agreement could be even improved if the assumption of equal constants of binding for all counterions was not made or if the existence of more than one set of independent binding sites $^{50,64}$ was assumed. Nevertheless, in order to get physically reasonable results which are consistent with our previous observations no such sidesteps from our understanding of the studied system were made.

As a part of the fitting procedure, the fractions of free counterions $f_{i}$ for every ionic species $i$ were calculated from the calorimetric model at each $r_{n}$. These fractions are shown in Fig. 9 together with the experimentally determined values. It can be seen that experimentally determined and model values

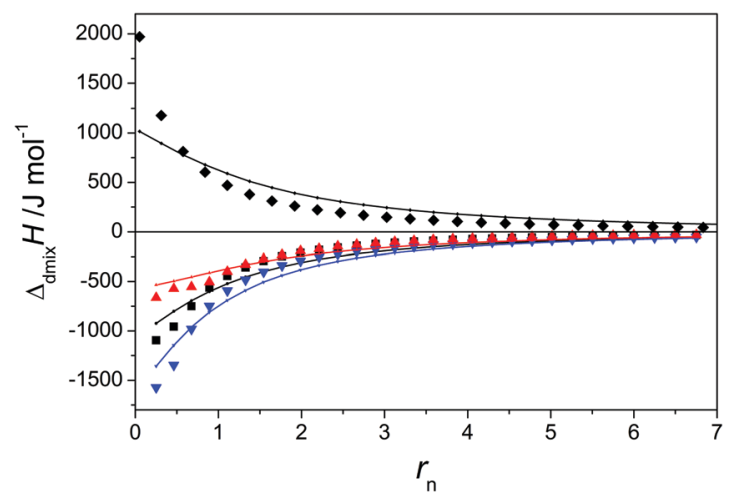

Fig. 8 Comparison of experimental ${ }^{33}$ (symbols) differential enthalpies of mixing, $\Delta_{\text {dmix }} H$, and results of global fitting (lines) for the titration of 0.005 monomol dm ${ }^{-3}$ PTALi with $0.15 \mathrm{~mol} \mathrm{dm}^{-3} \mathrm{CsCl}$ at $278.15 \mathrm{~K}, 298.15 \mathrm{~K}$, and $318.15 \mathrm{~K}$ (legend as in the previous figure) and for reverse titration (0.005 monomol dm ${ }^{-3}$ PTACs with $\left.0.15 \mathrm{~mol} \mathrm{dm}^{-3} \mathrm{LiCl}\right)$ at $298.15 \mathrm{~K}(\bullet)$ 


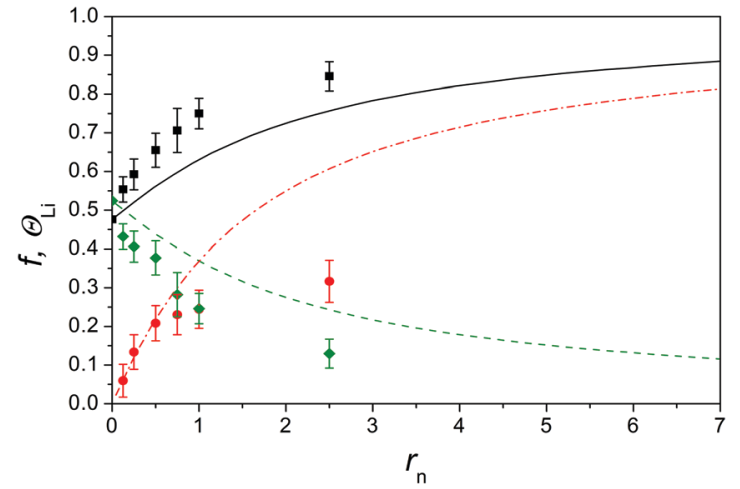

Fig. 9 Fractions of free and bound counterions as obtained from model analysis of calorimetric data for the titration of 0.005 monomol $\mathrm{dm}^{-3}$ PTALi with $0.15 \mathrm{~mol} \mathrm{dm}^{-3} \mathrm{NaCl}$ and $\mathrm{CsCl}$ at $298.15 \mathrm{~K}$. Model calculations: $f_{\mathrm{Li}}$ - continuous black line, $\theta_{\mathrm{Li}}$ - green dashed line, and $\theta_{\text {add }}$ - red dashdotted line. Experimental data: $f_{\mathrm{Li}}-\mathbf{\square}, \theta_{\mathrm{Li}}-\bullet$, and $\theta_{\text {add }}-\bigcirc$ (calculated as an average of values measured for the addition of $\mathrm{NaCl}$ and $\mathrm{CsCl}$ ).

of $f$ coincide at $r_{n}=0$, which is a direct consequence of the fact that $K_{i}$ of alkali metal counterions was estimated from $f_{\mathrm{Li}}$ in PTALi solution at $r_{n}=0$. At higher values of $r_{n}$, the concordance between measured and modelled $f$ is poorer although it is still reasonably good. As a consequence, the use of $K_{i}$ at larger $r_{n}$ values underestimates $f$ which consequently leads to the overestimation of $\theta$.

On the other hand, the existence of different kinds of binding sites ${ }^{64}$ cannot be completely discarded although no such evidence exists. It is quite possible that some counterions are non-specifically bound in the electrostatic atmosphere while the other ones may be bound by site-binding. ${ }^{65}$ In such a case it cannot be expected that the model which takes into account only one binding mode would successfully describe the observed mixing process.

The thermodynamic parameters, obtained during global fitting of the model function to the experimental data shown in Fig. 7 and 8, are graphically represented in Fig. 10 for $T=298.15 \mathrm{~K}$.

Taking into account coulombic interactions only, the addition of simple salt to PE salt solution should be an endothermic process. ${ }^{63}$ For the system under study, where the strongly hydrated lithium counterion was present both in the PE and in the added salt solutions, it was observed that the exchanged heat qualitatively agrees with the theoretical prediction assuming only Coulombic interactions. ${ }^{33}$ The mixing was notably more endothermic than expected when the PE salt included the weakly hydrated caesium counterion and $\mathrm{LiCl}$ was added whereas the process was strongly exothermic when lithium PE salt was mixed with $\mathrm{CsCl}$. It is therefore not surprising that thermodynamic analysis gave (at $298.15 \mathrm{~K}$ ) a slightly positive value for $\Delta_{\mathrm{b}} H_{\mathrm{Li}}^{\ominus}$ while $\Delta_{\mathrm{b}} H_{\mathrm{Na}}^{\ominus}$ and $\Delta_{\mathrm{b}} H_{\mathrm{CS}}^{\ominus}$ were negative: the latter more than the former. The extent of hydration of alkali ions decreases with increasing crystallographic radius of the ion and accordingly, hydration is becoming less and less exothermic from lithium to caesium. ${ }^{66}$ Although $\Delta_{\mathrm{b}} H^{\ominus}$ is small (in absolute sense) in comparison with $\Delta_{\mathrm{b}} G^{\ominus}$, values of standard molar enthalpies
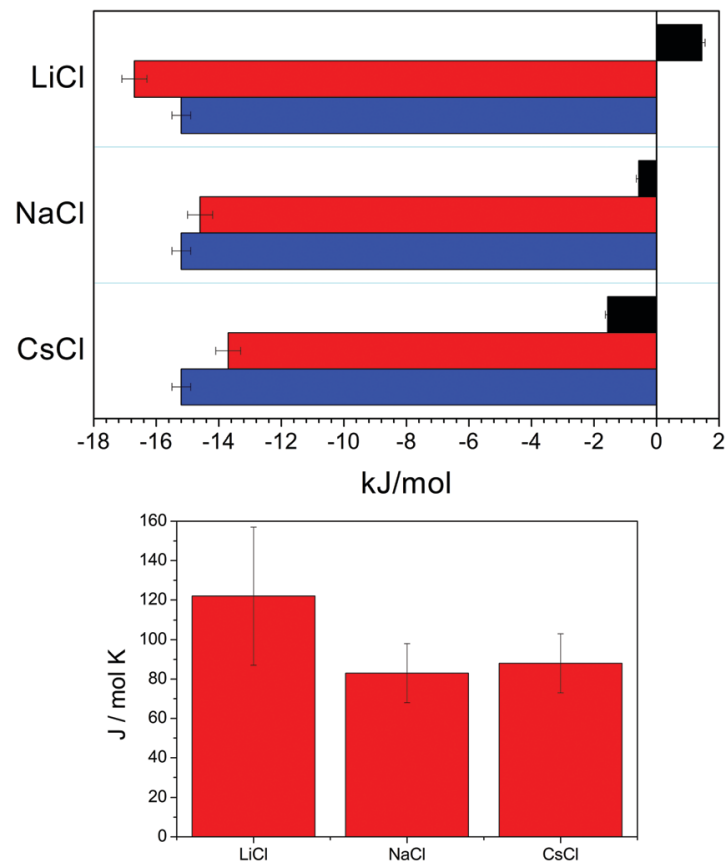

Fig. 10 Model parameters for binding of studied alkali metal counterions to the PTA ${ }^{-}$polyion at $298.15 \mathrm{~K}$. In the upper chart values of $\Delta_{b} H^{\ominus}$, $-T \Delta_{b} S^{\ominus}$, and $\Delta_{b} G^{\ominus}$ at $298.15 \mathrm{~K}$ are represented by black, red, and blue horizontal columns for binding of lithium, sodium, and caesium counterions to PTA ${ }^{-}$, respectively. Values of $\Delta_{\mathrm{b}} C_{p}^{\ominus}$ are plotted as vertical columns in the lower chart.

are measured rather accurately (see error bars in Fig. 10) so that the dependence of $\Delta_{\mathrm{b}} \mathrm{H}^{\ominus}$ on the kind of counterion can be firmly determined. If we recall Fig. S3 in the ESI, $\dagger$ where the total fraction of occupied binding sites on $\mathrm{PTA}^{-}$(i.e. the total fraction of polyion charge neutralised by bound counterions) is plotted, the mixing seems to be reduced to a process where upon mixing the bound counterion is just replaced by a counterion of the other kind.

During the binding of a counterion to a polyion some water molecules are released from the solvation shell of the counterion $^{67,68}$ (and usually also from the solvation shell of the polyion) to the bulk. The release of water molecules is energetically less favourable for counterions having more negative standard enthalpies of hydration - in our case this would apply to lithium counterions. This is reflected in $\Delta_{\mathrm{b}} H^{\ominus}$ calculated, where the highest $\Delta_{\mathrm{b}} H^{\ominus}$ was obtained for lithium and the lowest one for caesium.

By fixing $\Delta_{\mathrm{b}} G^{\ominus}$ to be equal for all alkali counterions, the dependence of $\Delta_{\mathrm{b}} H^{\ominus}$ on the kind of counterion also determines such a dependence of $\Delta_{\mathrm{b}} S^{\ominus}$. The increase of $\Delta_{\mathrm{b}} S^{\ominus}$ is the highest for strongly hydrated lithium counterions, whereas it is the smallest for caesium counterions. The entropy increase can be explained by the release of water molecules. Namely, one can expect that the ion having more water molecules in its solvation shell will lose more water molecules upon binding than the ion that is poorly hydrated. The release of more water molecules from the solvation shell to bulk water is accompanied by a higher increase of entropy. When values of $\Delta_{\mathrm{b}} H^{\ominus}$ and 
$-T \cdot \Delta_{\mathrm{b}} S^{\ominus}$ are compared, it is evident that the contribution to $\Delta_{\mathrm{b}} G^{\ominus}$ stems almost entirely from the product $-T \cdot \Delta_{\mathrm{b}} S^{\ominus}$. Obviously, the increase of entropy is a driving force of the studied process which is an observation, which was in similar cases often quoted also in the literature. ${ }^{50,68,69}$

The assumption that the binding of counterions to the polyion is accompanied by the partial dehydration of counterions is also corroborated by changes in $\Delta_{\mathrm{b}} C_{p}^{\ominus}$. Fig. 10 shows that changes of $\Delta_{\mathrm{b}} C_{p}^{\ominus}$ do not show any obvious dependence on the counterion size. However, the changes in $\Delta_{\mathrm{b}} C_{p}^{\ominus}$ upon counterion binding are positive for all the counterions studied. The increase of $\Delta_{\mathrm{b}} C_{p}^{\ominus}$ may be in our case explained by the increased fluctuations of the enthalpy of the system. ${ }^{70}$ Namely, the gap between different energy levels accessible to water molecules being part of the ionic hydration shell is large and thus the fluctuations in enthalpy are relatively small. When a water molecule is released into the bulk phase, the differences between enthalpic levels accessible to the water molecule become smaller. Consequently, the fluctuations in enthalpy are increased, which leads to an increased value of $\Delta_{\mathrm{b}} C_{p}^{\ominus}$.

The positive values of $\Delta_{\mathrm{b}} C_{p}^{\ominus}$ therefore confirm our premise that some water molecules are released upon counterion binding. These results are in good agreement with similar treatment of calorimetric data for some other polyelectrolytes. ${ }^{50,51}$

A similar analysis of titration curves as performed for mixing PTALi with alkali chlorides was tried to be carried out also for mixing PTALi with tetraalkylammonium chlorides. Considering the different nature of tetraalkylammonium ions, in addition to a fit with one set of binding sites we also tried a fit with two sets of binding sites but in both cases analysis failed to give any reasonable result. This was yet another indication that in the case of tetraalkylammonium counterions besides electrostatics other interactions are at play and that a more elaborate model should be used for obtaining standard thermodynamic parameters.

\section{Conclusions}

The binding of counterions to the conjugated polyion, observed during the mixing aqueous solution of lithium salt of poly(thiophen3-ylacetic acid) (PTALi) with alkali metal chlorides at $25{ }^{\circ} \mathrm{C}$, can be satisfactorily described by electrostatic theories. While none of the alkali metal counterions seems to bind preferentially, the mixing of tetraalkylammonium $\left(\mathrm{TAA}^{+}\right)$chlorides with PTALi showed that $\mathrm{TAA}^{+}$cations with longer alkyl chains bind to the polyion more extensively than predicted by electrostatics. The fraction of bound counterions increases in the order from the tetramethylammonium to tetrabutylammonium counterion and is in the case of tetrapropylammonium and tetrabutylammonium ions accompanied by the blue shift in the UV/vis absorption spectrum. The increase of the diffusion coefficient $D_{\mathrm{p}}$ of the polyion was observed in all cases when alkali metal or tetraalkylammonium chlorides were added to PTALi but the increase of $D_{\mathrm{p}}$ was again more pronounced when tetrapropylammonium and tetrabutylammonium chloride solutions were added, suggesting that the addition of these counterions promotes further transition of $\mathrm{PTA}^{-}$polyions to more compact conformation.

Thermodynamic analysis of calorimetric data obtained upon mixing PTALi solution with solutions of alkali metal chlorides confirmed our assumption that observed differences in heats of mixing are a consequence of different strengths and extents of hydration of these cations. The calculated standard enthalpies of binding of alkali metal counterions clearly indicate that dehydration of these counterions (release of water molecules from the counterion hydration shell into the bulk water) takes place upon binding. Namely, while $\Delta_{\mathrm{b}} H^{\ominus}$ is positive for $\mathrm{Li}^{+}$, the same quantity is increasingly negative from $\mathrm{Na}^{+}$to $\mathrm{Cs}^{+}$which is in concordance with the finding that the hydrogen bond in the first hydration shell of $\mathrm{Li}^{+}$is stronger than the one in the bulk water and, vice versa, hydrogen bonds in hydration shells of $\mathrm{Na}^{+}$and (especially) $\mathrm{Cs}^{+}$are weaker than those in the bulk water. ${ }^{71,72}$ On the other side, the thermodynamic model, despite including an additional kind of binding site, completely failed in describing mixing of PTALi with tetraalkylammonium chloride solutions. This is yet another evidence that the nature of binding of tetraalkylammonium counterions to the PTA ${ }^{-}$ polyion is different from the one observed for alkali counterions and that it may include additional interactions, which are most probably of hydrophobic nature. Further theoretical studies based on molecular dynamics and experiments exploiting the nuclear Overhauser effect between the ions and the polyelectrolyte might be of help in resolving different modes of binding of both kinds of counterions to $\mathrm{PTA}^{-}$.

\section{Acknowledgements}

This study was supported by the Slovenian Research Agency fund (ARRS) through the Program 0103-0201. The use of instruments at the NMR Spectroscopy Unit of the Research Infrastructure Centre UL FCCT while performing preliminary measurements is acknowledged. G. H. was supported by the National Grant for young researchers and by Slovene human resources development and scholarship fund. Prof. Dr Vojko Vlachy (University of Ljubljana) is acknowledged for valuable discussion through this work. The authors also thank Isabelle Correia (Laboratoire des Biomolécules) and Francois Ribot (Laboratoire de la Chimie de la Matière Condensée de Paris) for the access to the $500 \mathrm{MHz}$ and the $300 \mathrm{MHz}$ NMR spectrometers and fruitful discussions.

\section{References}

1 Y.-J. Cheng, S.-H. Yang and C.-S. Hsu, Chem. Rev., 2009, 109, 5868-5923.

2 Z. He, C. Zhong, X. Huang, W.-Y. Wong, H. Wu, L. Chen, S. Su and Y. Cao, Adv. Mater., 2011, 23, 4636-4643.

3 Y.-M. Chang, R. Zhu, E. Richard, C.-C. Chen, G. Li and Y. Yang, Adv. Funct. Mater., 2012, 22, 3284-3289.

4 H.-J. Wang, C.-P. Chen and R.-J. Jeng, Materials, 2014, 7, 2411-2439. 
5 J. H. Burroughes, D. D. C. Bradley, A. R. Brown, R. N. Marks, K. Mackay, R. H. Friend, P. L. Burn and A. B. Holmes, Nature, 1990, 347, 539-541.

6 V. Cimrová, W. Schmidt, R. Rulkens, M. Schulze, W. Meyer and D. Neher, Adv. Mater., 1996, 8, 585-588.

7 A. F. Thünemann and D. Ruppelt, Langmuir, 2001, 17, 5098-5102.

8 F. Huang, H. Wu, D. Wang, W. Yang and Y. Cao, Chem. Mater., 2004, 16, 708-716.

9 Z. Gu, Y.-J. Bao, Y. Zhang, M. Wang and Q.-D. Shen, Macromolecules, 2006, 39, 3125-3131.

10 P. Bäuerle and S. Scheib, Adv. Mater., 1993, 5, 848-853.

11 I. Levesque and M. Leclerc, Chem. Commun., 1995, 2293-2294.

12 R. D. McCullough and S. P. Williams, Chem. Mater., 1995, 7, 2001-2003.

13 M. J. Marsella and T. M. Swager, J. Am. Chem. Soc., 1993, 115, 12214-12215.

14 A. Boldea, I. Levesque and M. Leclerc, J. Mater. Chem., 1999, 9, 2133-2138.

15 S. Bernier, S. Garreau, M. Béra-Abérem, C. Gravel and M. Leclerc, J. Am. Chem. Soc., 2002, 124, 12463-12468.

16 I.-B. Kim, A. Dunkhorst, J. Gilbert and U. H. F. Bunz, Macromolecules, 2005, 38, 4560-4562.

17 Z. Zhang, Q. Fan, P. Sun, L. Liu, X. Lu, B. Li, Y. Quan and W. Huang, Macromol. Rapid Commun., 2010, 31, 2160-2165.

18 L. Chen, D. W. McBranch, H.-L. Wang, R. Helgeson, F. Wudl and D. G. Whitten, Proc. Natl. Acad. Sci. U. S. A., 1999, 96, 12287-12292.

19 A. Satrijo and T. M. Swager, J. Am. Chem. Soc., 2007, 129, 16020-16028.

20 F. Chu, G. Tsiminis, N. A. Spooner and T. M. Monro, Sens. Actuators, B, 2014, 199, 22-26.

21 H.-A. Ho, M. Béra-Abérem and M. Leclerc, Chem. - Eur. J., 2005, 11, 1718-1724.

22 A. V. Ambade, B. S. Sandanaraj, A. Klaikherd and S. Thayumanavan, Polym. Int., 2007, 56, 474-481.

23 M. Béra-Abérem, H.-A. Ho and M. Leclerc, Tetrahedron, 2004, 60, 11169-11173.

24 X. Liu, Q. Fan and W. Huang, Biosens. Bioelectron., 2011, 26, 2154-2164.

25 R. Yang, H. Wu, Y. Cao and G. C. Bazan, J. Am. Chem. Soc., 2006, 128, 14422-14423.

26 D. K. Kim, S. W. Han, C. H. Kim, J. D. Hong and K. Kim, Thin Solid Films, 1999, 350, 153-160.

27 O. Mermut, J. Lefebvre, D. G. Gray and C. J. Barrett, Macromolecules, 2003, 36, 8819-8824.

28 R. D. McCullough, P. C. Ewbank and R. S. Loewe, J. Am. Chem. Soc., 1997, 119, 633-634.

29 B.-S. Kim, L. Chen, J. P. Gong and Y. Osada, Macromolecules, 1999, 32, 3964-3969.

30 P. Vallat, J.-M. Catala, M. Rawiso and F. Schosseler, Macromolecules, 2007, 40, 3779-3783.

31 I. E. Franco, P. Lorchat, J.-P. Lamps, M. Schmutz, A. Schröder, J.-M. Catala, J. Combet and F. Schosseler, Langmuir, 2012, 28, 4815-4828.
32 G. Hostnik, D. Bondarev, J. Vohlídal, S. Čebašek, E. Žagar, V. Vlachy and J. Cerar, J. Mol. Liq., 2014, 198, 173-180.

33 G. Hostnik, V. Vlachy, D. Bondarev, J. Vohlídal and J. Cerar, Phys. Chem. Chem. Phys., 2015, 17, 2475-2483.

34 K. Collins, Biophys. J., 1997, 72, 65-76.

35 I. Furó, J. Mol. Liq., 2005, 117, 111-137.

36 S. Dengler, A. Klaus, G. J. T. Tiddy and W. Kunz, Faraday Discuss., 2013, 160, 121-133.

37 S. Dengler, G. J. T. Tiddy, L. Zahnweh and W. Kunz, Colloids Surf., A, 2014, 457, 414-418.

38 P. Stilbs and B. Lindman, J. Magn. Reson., 1982, 48, 132-137.

39 R. Rymdén and P. Stilbs, J. Phys. Chem., 1985, 89, 2425-2428.

40 G. Hostnik, V. Vlachy, D. Bondarev, J. Vohlídal and J. Cerar, Acta Chim. Slov., 2012, 59, 571-581.

41 G. Hostnik, V. Vlachy, D. Bondarev, J. Vohlídal and J. Cerar, Acta Chim. Slov., 2012, 59, 582-589.

42 A. Krȩżel and W. Bal, J. Inorg. Biochem., 2004, 98, 161-166.

43 C. S. Johnson, Jr., Prog. Nucl. Magn. Reson. Spectrosc., 1999, 34, 203-256.

44 D. Wu, A. Chen and C. Johnson, J. Magn. Reson., 1995, 115, 260-264.

45 P. T. Callaghan and D. N. Pinder, Macromolecules, 1983, 16, 968-973.

46 P. T. Callaghan and J. Lelievre, Biopolymers, 1985, 24, 441-460.

47 A. Katchalsky, Pure Appl. Chem., 1971, 26, 327-374.

48 D. Dolar, in Polyelectrolytes, ed. E. Sélégny, M. Mandel, U. P. Strauss and D. Reidel, Dordrecht, Netherlands, 1st edn, 1974, vol. 1, pp. 97-113.

49 J. Piñero, L. B. Bhuiyan, J. Reščič and V. Vlachy, Acta Chim. Slov., 2008, 55, 521-527.

50 M. Bončina, J. Lah, J. Reščič and V. Vlachy, J. Phys. Chem. B, 2010, 114, 4313-4319.

51 M. Bončina, M. Lukšič, M. Seručnik and V. Vlachy, Mol. Phys., 2014, 112, 1222-1229.

52 J. Lah, N. M. Maier, W. Lindner and G. Vesnaver, J. Phys. Chem. B, 2001, 105, 1670-1678.

53 J. Lah, I. Drobnak, M. Dolinar and G. Vesnaver, Nucleic Acids Res., 2008, 36, 897-904.

54 K. D. Collins, Methods, 2004, 34, 300-311.

55 N. Vlachy, B. Jagoda-Cwiklik, R. Vácha, D. Touraud, P. Jungwirth and W. Kunz, Adv. Colloid Interface Sci., 2009, 146, 42-47.

56 F. J. M. Schipper, K. Kassapidou and J. C. Leyte, J. Phys.: Condens. Matter, 1996, 8, 9301.

57 S. Förster, M. Schmidt and M. Antonietti, Polymer, 1990, 31, 781-792.

58 L. Wang, M. M. Garner and H. Yu, Macromolecules, 1991, 24, 2368-2376.

59 P. Vallat, J. M. Catala, M. Rawiso and F. Schosseler, Europhys. Lett., 2008, 82, 28009.

60 K. Faïd, M. Frechette, M. Ranger, L. Mazerolle, I. Levesque, M. Leclerc, T.-A. Chen and R. D. Rieke, Chem. Mater., 1995, 7, 1390-1396. 
61 J. L. Brédas, G. B. Street, B. Thémans and J. M. André, J. Chem. Phys., 1985, 83, 1323-1329.

62 M. Bernardi, M. Giulianini and J. C. Grossman, ACS Nano, 2010, 4, 6599-6606.

63 G. E. Boyd, D. P. Wilson and G. S. Manning, J. Phys. Chem., 1976, 80, 808-810.

64 G. Scatchard, J. S. Coleman and A. L. Shen, J. Am. Chem. Soc., 1957, 79, 12-20.

65 I. Rouzina and V. A. Bloomfield, Biophys. Chem., 1997, 64, 139-155.

66 Y. Marcus, J. Chem. Soc., Faraday Trans., 1987, 83, 339-349. 72 J. Mähler and I. Persson, Inorg. Chem., 2012, 51, $425-438$.
67 M. Satoh and J. Komiyama, Polymeric Materials Encyclopedia, CRC Press, Inc., Boca Raton, Florida, 1st edn, 1996, vol. 8, pp. 5807-5816.

68 C. G. Sinn, R. Dimova and M. Antonietti, Macromolecules, 2004, 37, 3444-3450.

69 I. Pochard, A. Foissy and P. Couchot, Colloid Polym. Sci., 1999, 277, 818-826.

70 K. A. Sharp and B. Madan, J. Phys. Chem. B, 1997, 101, 4343-4348. 71 J. Stangret and T. Gampe, J. Phys. Chem. A, 2002, 106, 5393-5402. 